\title{
Studies on cut-off grinding of BK7 optical glass using thin diamond wheels
}

\author{
S.Y. Luo ${ }^{\text {a,* }}$, Y.Y. Tsai ${ }^{\text {b }}$, C.H. Chen ${ }^{\text {a }}$ \\ a Department of Mechatronic Engineering, Huafan University, Shihtin, Taipei, Taiwan \\ ${ }^{\mathrm{b}}$ Department of Mechanical Engineering, National Taiwan University, Taipei, Taiwan
}

Received 17 August 2004; accepted 14 November 2005

\begin{abstract}
This paper investigated the characteristics and the wear modes of the diamond grits in the cut-off grinding BK7 glass using thin diamond wheel. The grinding forces, the grinding ratio, the width of cut, the straightness of cut and chipping produced during the cut-off grinding were also studied.

Experimental results showed that the major wear process of the diamond particles observed for a lower transverse velocity during the cutting-off BK7 glass was from protrusive, partial breakage, pulled-out, to new conditions, which obtain a freer cutting ability, a better grinding ratio and a better straightness of cut. However, when a too high transverse velocity was used, the diamond wear pattern displayed some macro-breakage and flatten conditions occurred on the worn wheel surface, thereby causing the unstable grinding forces, a low grinding ratio and a poor straightness of cut. Besides, the chipping condition of the bottom on the edge of the kerf during the cut-off grinding of BK7 glass was larger than that of the top, which is due to the down-mode grinding.
\end{abstract}

(C) 2006 Elsevier B.V. All rights reserved.

Keywords: Cut-off grinding; BK7 glass; Thin diamond wheel; Wear

\section{Introduction}

Optical lens in the optic industries are very important components that are widely used to the applications of digital cameras, scanner, projector, etc. These glasses for lens during the fabrication are usually cut-off into a die by a slicing machine using thin diamond grinding wheels. Due to the brittleness of the glasses, the chippings on the cutting edges of the glasses are always occurred and the straightness of cut is also concerned. In addition, the wear and deflection of the thin diamond wheels often cause a limited blade life and chipping of glasses.

In order to improve the performance for the cut-off grinding of the hard brittle materials, some studies have been conducted. Yamasaka et al. [1] indicated that the straightness of the sliced surface in precision cut-off grinding of alumina ceramics with thin diamond wheels strongly correlates with the axial deflection of a thin grinding wheel, which is caused by the side forces acting on the side surface of the grinding wheel. As a result, the straight-

\footnotetext{
* Corresponding author. Tel.: +886 226632102x4018; fax: +8862 26631119. E-mail address: syluo@huafan.hfu.edu.tw (S.Y. Luo).
}

ness can be improved remarkably according to decreasing the side force both by decreasing the depth of cut and increasing the table speed with retaining a stock removal rate, and by improving the flatness on the side surface of the grinding wheel.

Ohbuchi et al. [2] stated that the warp of workpiece for high precision cut-off grinding of $\mathrm{Al}_{2} \mathrm{O}_{3}$ - TiC ceramic with metalbonded thin diamond wheels was caused due to wheel deflection by grinding forces because of its low stiffness. The displacement of slot produced increased with increasing normal grinding force, but it can be restrained by using large mesh size wheels because of lower grinding forces.

Miwa and Inasaki [3] indicated that the dicing force on silicon wafer dicing increased in case of high feed rate, high depth of cut, low blade speed and the down cutting mode. The diamond blade wear and the chipping of groove edges produced increased as the dicing force increased.

Even though several studies on the performance of the grinding hard and brittle materials had been investigated [4-11]. However, studies relating the thin diamond wheels on the cut-off grinding of a thick BK7 glass were little reported. In this paper, the wear of the thin diamond wheels and the cut quality of the thick optical glass were studied. 


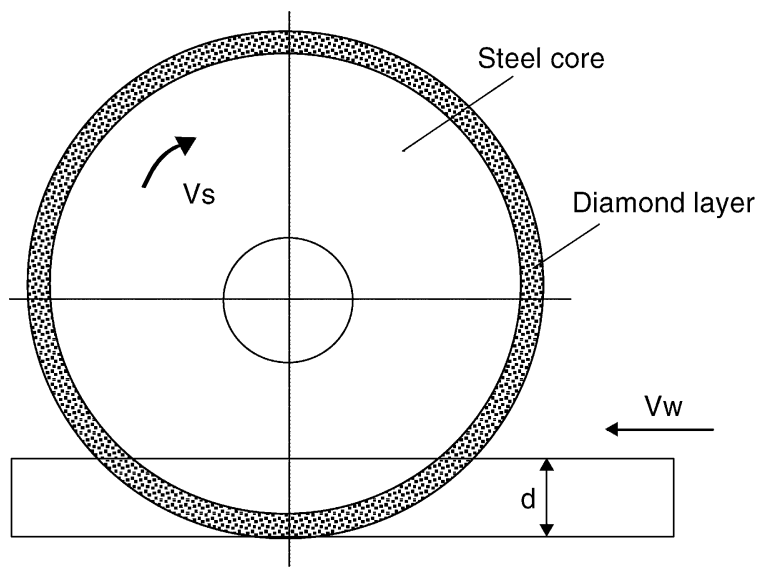

Fig. 1. Geometry in the cut-off grinding.

\section{Experimental procedure}

Cut-off grinding tests of BK7 glass using a thin metal-bonded diamond wheel were performed on a precision surface grinder, its engagement kinematics and down-mode grinding being shown in Fig. 1. The outer diameter of the wheel was $150 \mathrm{~mm}$ and the thickness of the rim was $0.8 \mathrm{~mm}$. The overhang length of the thin wheel was $40 \mathrm{~mm}$. The metal bond of a diamond wheel was mainly bronze. The diamond of 100/120 US mesh was used at 35 concentration $\left(1.54\right.$ carats $\left./ \mathrm{cm}^{3}\right)$. The depth of cut-off of BK7 glass workpieces was $10 \mathrm{~mm}$. The cut-off grinding conditions in the tests are given in Table 1.

For each test, the vertical and horizontal grinding forces were measured by means of a quartz piezoelectric type dynamometer (KISTLER type 9257B) connected to an A/D converter and a personal computer, which in turn, calculates the normal and tangential forces. Besides, the radial wear of the wheels was measured by means of a toolmaker's microscope. A characteristic grinding ratio (the ratio of the volume of material removed per unit volume of wheel wear) was used to investigate the performance of the wheels at several cut-off grinding conditions. The worn diamond conditions on the wheel surface were traced and analyzed using a CCD, stereo optical microscope and SEM.

Furthermore, the straightness, width and chipping of the sliced surface were examined using a toolmaker's microscope and SEM. Surface roughness of the BK7 glass after cut-off grinding was examined using a profilometer.

\section{Experimental results}

\subsection{Diamond wear conditions}

Diamond particle wear after cut-off grinding BK7 glass was examined by counting the worn diamond abrasives on the wheel surface using a stereomicroscope. The worn diamond particle conditions in the tests can be broadly classified as follows [9]: (a) protrusive particle, (b) flattened particle, (c) breakage particle,

Table 1

Cut-off grinding conditions

\begin{tabular}{ll}
\hline Cutting mode & Down cutting \\
\hline Spindle speed $(\mathrm{rpm})$ & 3500 \\
Wheel velocity, $V_{\mathrm{s}}(\mathrm{m} / \mathrm{s})$ & 27.5 \\
Transverse velocity, $V_{\mathrm{w}}(\mathrm{mm} / \mathrm{min})$ & $50,100,150$ \\
Depth of cut, $d(\mathrm{~mm})$ & 10 \\
Cutting fluid & Water \\
Workpiece (size) & BK7 glass $(140 \mathrm{~mm} \times$ \\
& $100 \mathrm{~mm} \times 10 \mathrm{~mm})$ \\
Workpiece section being ground & $140 \mathrm{~mm} \times 10 \mathrm{~mm}$ \\
\hline
\end{tabular}

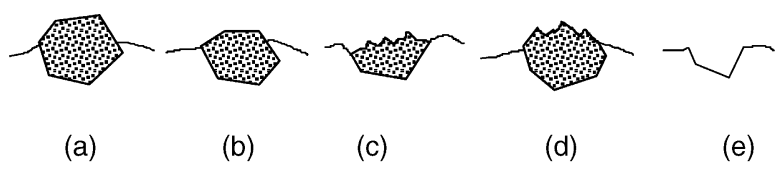

Fig. 2. Scheme of wear modes of the diamond abrasive condition: (a) protrusive particle, (b) flattened particle, (c) breakage particle, (d) partial breakage particle and (e) pull-out hole.

(d) partial breakage particle and (e) pull-out hole. Fig. 2 shows schematically the wear modes of these diamond particles.

The diamond wear variations on the wheel surface for some diamond particles traced by a CCD and stereo microscope with the cut-off area of BK7 glass are shown in Fig. 3a-c under three different transverse velocities, respectively. The color differences in Fig. 3 represent the wear modes of the diamond particles during the cut-off of the BK7 glass. The lighter color shows the more protrusion of the diamond grit with a better cutting ability. Conversely, the darker color represents the larger breakage or flatten particle occurred on the worn wheel surface, thereby causing a poorer cutting behavior. When the color in the figure is black, the diamond particle represents a pulled-out state. From Fig. 3a, it can be seen that the good protrusive diamond particles (type a) for the grit numbers 16 and 17 at the slower transverse velocity of $V_{\mathrm{w}}=50 \mathrm{~mm} / \mathrm{min}$ can maintain a longer cutting ability (the light color zone is larger). For the diamond grit numbers $12-14$, when the amount of cut-off area reaches to about $16.5 \mathrm{~mm} \times 4500 \mathrm{~mm}$, the diamond particles on the worn wheel surface start to produce the partial breakage, then breakage or flat and, shortly, the pull-out condition. Besides, most of the good diamond grits on the worn surface during the cut-off grinding produce the partial breakage condition (type c), which in turn, be plunked out of the bond. They seldom develop into flattened or macro-breakage conditions. This implies that the diamond wheel during the cut-off grinding of BK7 glass would be free cutting behavior, thereby forming a better straightness of cut and a smaller chipping of the cutting edge. Hence, the working mechanism of the diamond particles at a lower transverse velocity during the cut-off grinding of BK7 glass was predicted that the good grains were initially subjected to a lower cyclic stress to maintain a long time and the metal bond wore rapidly to make the grit to produce more protrusion, thereby causing the grains to suffer the larger force to produce the partial breakage, then fast pulled-out of the bond. Furthermore, the new particles (the grit numbers 19-34) on the worn wheel surface during the cut-off grinding would be constantly protrusive, which make the amount of the protrusive diamond grits to maintain a high percentage (the light color zone) with the cut-off area of BK7 glass, hence generating a good redressing ability of the thin diamond wheel. This can improve its steady cutting action.

When the transverse velocity of $V_{\mathrm{w}}=100 \mathrm{~mm} / \mathrm{min}$ was employed (Fig. 3b), some good protrusive diamond particles (the grit numbers 16-22) with the cut-off area of BK7 glass can maintain a long time. Some good protrusive particles (the grit numbers 3-15 and 24-26) under a faster transverse velocity were subjected to a higher load, thereby leading the grits to be easily fractured. This causes the diamond wheel to display a proper working life. Furthermore, when the more transverse velocity of 

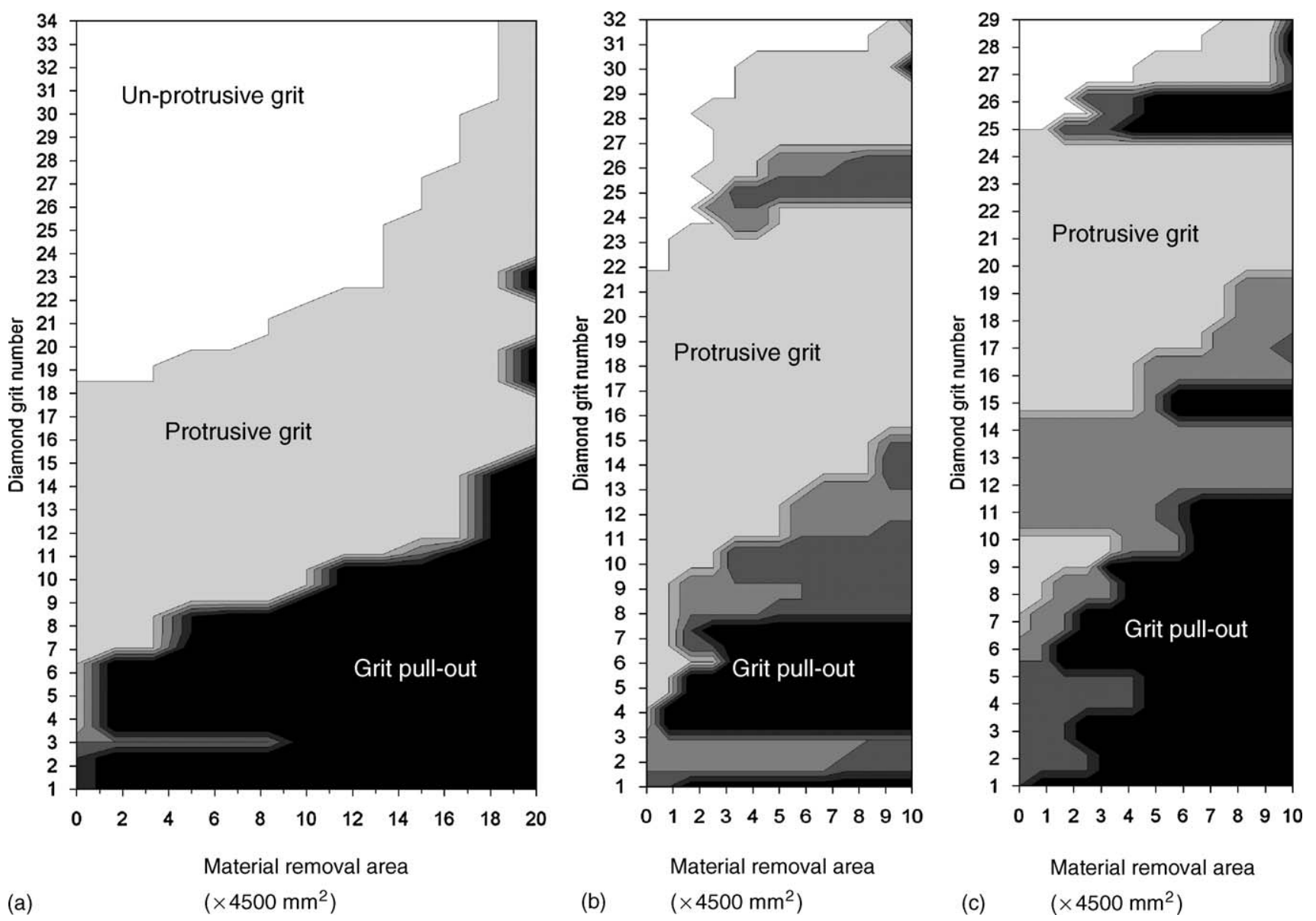

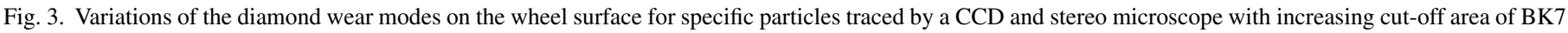

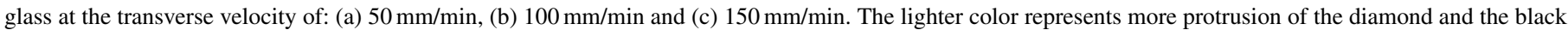
color shows the pull-out of the grits.

$V_{\mathrm{w}}=150 \mathrm{~mm} / \mathrm{min}$ was used (Fig. $3 \mathrm{c}$ ), the good protrusive diamond particles (the grit numbers 7-10 and 15-19) on the worn wheel surface would quickly become the more partial breakage and macro-breakage conditions, which have a large amount of breakage particles on the worn surface. Besides, the number of the new diamond particles produced on the bond displays less than those of the breakage and pull-out particles. This makes the effective cutting particles to become the fewer amounts on the bond at the end of cut-off grinding (the light color zone decreases with the increase of the cut-off area in Fig. 3c). It can be found that the new protrusive diamonds (the grit numbers 25-29) during the grinding faster produce the breakage and pull-out conditions. These diamond behaviors would cause the wheel to become glazing during the cut-off grinding of BK7 glass. It implies that the diamond wheel must be redressed to restore its cutting ability.

The percentage of each wear mode of diamond particle (see Fig. 2) counted on the worn surface with the increasing of the material removal area at the transverse velocity of 50, 100 and $150 \mathrm{~mm} / \mathrm{min}$ is shown in Fig. 4a-c, respectively. From Fig. 4a, it can be seen that some good protrusive particle (refer to the grit numbers 4-9 in Fig. 3a) at the initial cutting produce easily to pull out of the bond due to the unsteady diamond retention just after the dressing. Thereafter, the cutting behavior would reach to steady. It can be found that the percentage of protrusive particle with a better cutting ability and partial breakage abrasive on the worn wheel surface maintain about 35 and 10-15\%, respectively, and the percentage of pull-out particle on the worn wheel surface are about 50-55\%. In addition, the diamond particles on the worn surface rarely generate the breakage and flatten conditions, which lead the wheel to be dull. Hence, the diamond particle on the bond at the lower transverse velocity forms the following cycle: good protrusive condition, partial breakage condition, pull-out hole and new protrusive condition for cut-off BK7 glass, thereby generating a good redressing behavior.

When the transverse velocity of $V_{\mathrm{w}}=100 \mathrm{~mm} / \mathrm{min}$ was employed (Fig. 4b), with the increase of cut-off area of BK7 glass, the percentage of good protrusive diamond particle decreased to about $30 \%$ and the percentage of partial breakage particle increased to about $20 \%$. In addition, the percentage of breakage particle on the worn wheel surface displays about $10 \%$ while the percentage of pull-out hole on the worn wheel surface displays about $45 \%$. When the transverse velocity is increased to $150 \mathrm{~mm} / \mathrm{min}$ (Fig. 4c), it can be found that the percentage of protrusive diamond particle with a better cutting action on the bond become the fewer amounts of about $22 \%$ and the percentage of 


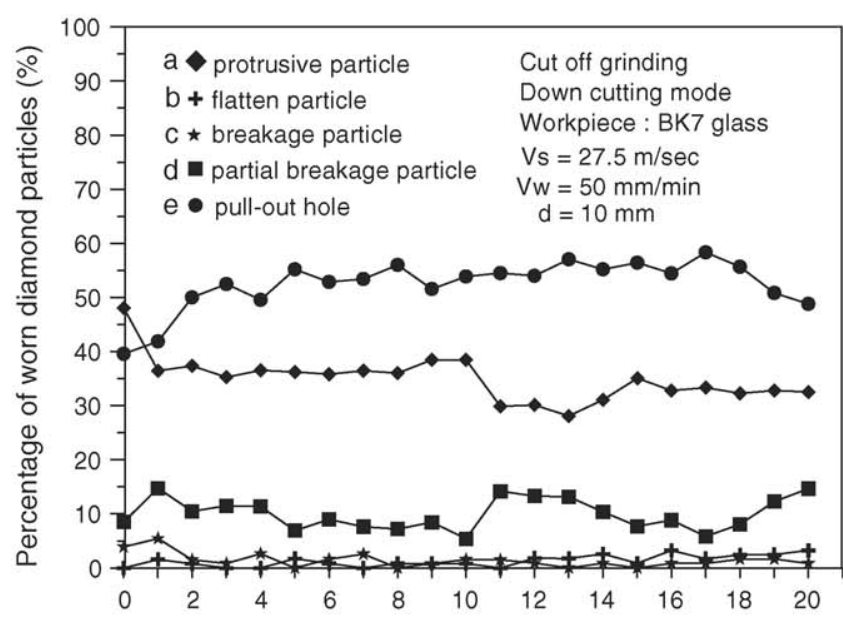

(a)

Material removal area $\left(X 4500 \mathrm{~mm}^{2}\right)$
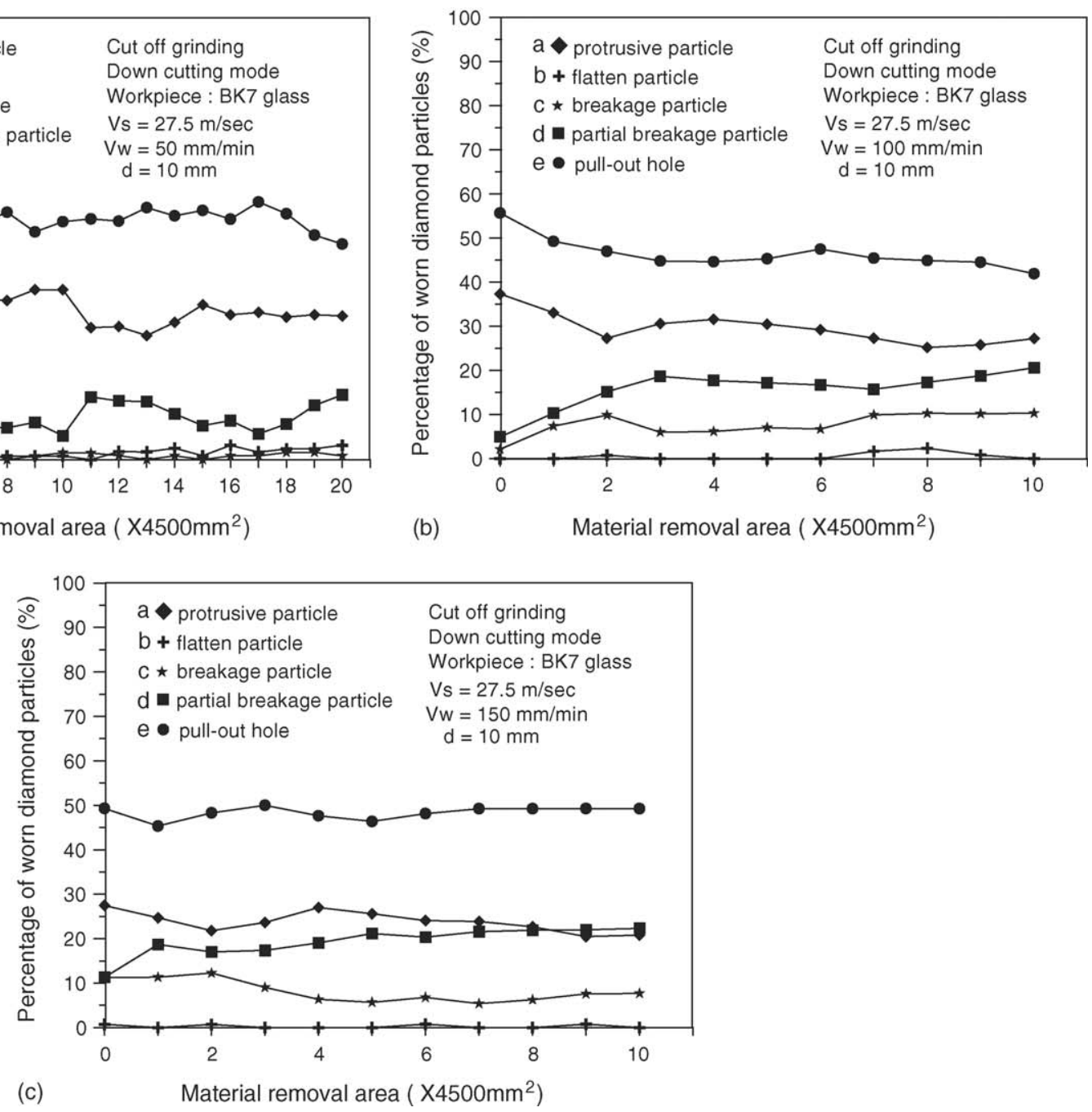

Fig. 4. Variations of the percentage of each wear mode of diamond particle counted on the worn surface with the increasing of the material removal area at the transverse velocity of: (a) $50 \mathrm{~mm} / \mathrm{min}$, (b) $100 \mathrm{~mm} / \mathrm{min}$ and (c) $150 \mathrm{~mm} / \mathrm{min}$.

partial breakage particle increases. In addition, the percentage of pull-out hole on the worn wheel surface displays about $50 \%$ and some breakage particle display on the worn wheel surface. This diamond wear pattern causes the effective cutting edges on the wheel surface to become the fewer amounts. Hence, the diamond particles at the higher transverse velocity suffer from the larger loads due to a larger depth of cut per grain during grinding, which produce the percentage of breakage grit condition to increase, thereby leading the diamond protrusive degree to decrease. This would make the wheel's cutting ability to become poor.

\subsection{Grinding ratio}

The radial wheel wear and the grinding ratio obtained during the cut-off grinding of BK7 glass are shown in Fig. 5 under three different transverse velocities. It can be seen that the radial wheel wear increases linearly with the increase of the cut-off area. The grinding radio obtained under the transverse velocity of 50 and $100 \mathrm{~mm} / \mathrm{min}$ is similar with 2461 and 2441, respectively. From the diamond wear pattern (see Figs. 3 and 4), the number of good

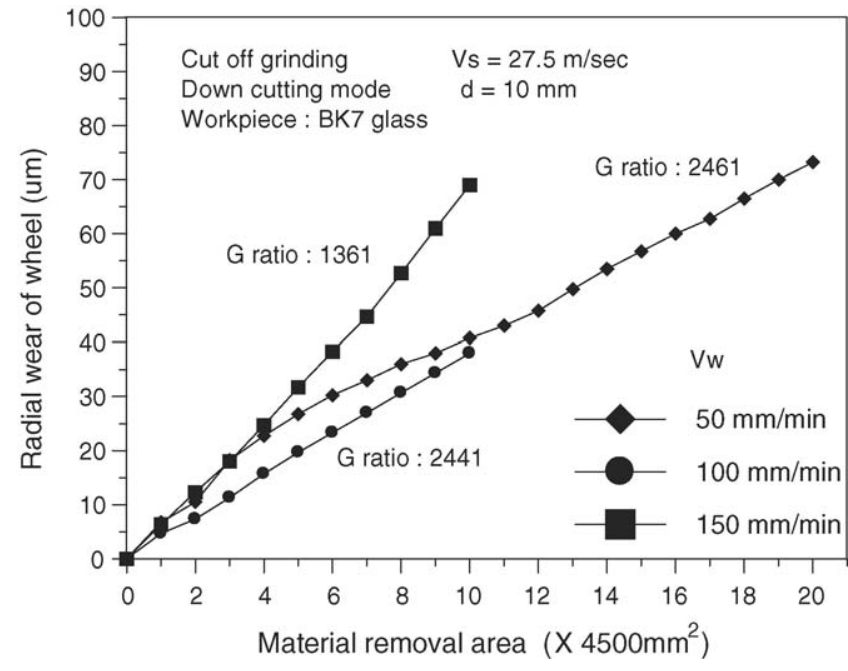

Fig. 5. The radial wheel wear and the grinding ratio obtained during the cut-off grinding of BK7 glass under three different transverse velocities. 
protrusive diamond particles and partial breakage particles on the worn wheel surface at the transverse velocity of $50 \mathrm{~mm} / \mathrm{min}$ is larger, hence producing a better cutting action. So, its wheel wear is smaller and the grinding ratio is larger a little. However, when the transverse velocity is increased to $150 \mathrm{~mm} / \mathrm{min}$, the diamond particles during grinding are subjected to a higher load. This would lead the grits to produce easily fracture and pull-out, which causes the wheel to wear fast. Hence, the grinding ratio of the wheel obtained is lower with 1361.

\subsection{Cut-off grinding forces}

The average axial grinding forces, $F_{\mathrm{a}}$, during the cut-off of BK7 glass with the material removal area are shown in Fig. 6a-c, and the average normal and tangential grinding forces, $F_{\mathrm{n}}$ and $F_{\mathrm{t}}$, produced are shown in Fig. 7a-c under three different transverse velocities, respectively. From Fig. 6a, it can be seen that the initial axial cut-off grinding forces produced at a slower transverse velocity of $50 \mathrm{~mm} / \mathrm{min}$ were smaller and more stable. Thereafter, the axial grinding forces produced a little increase

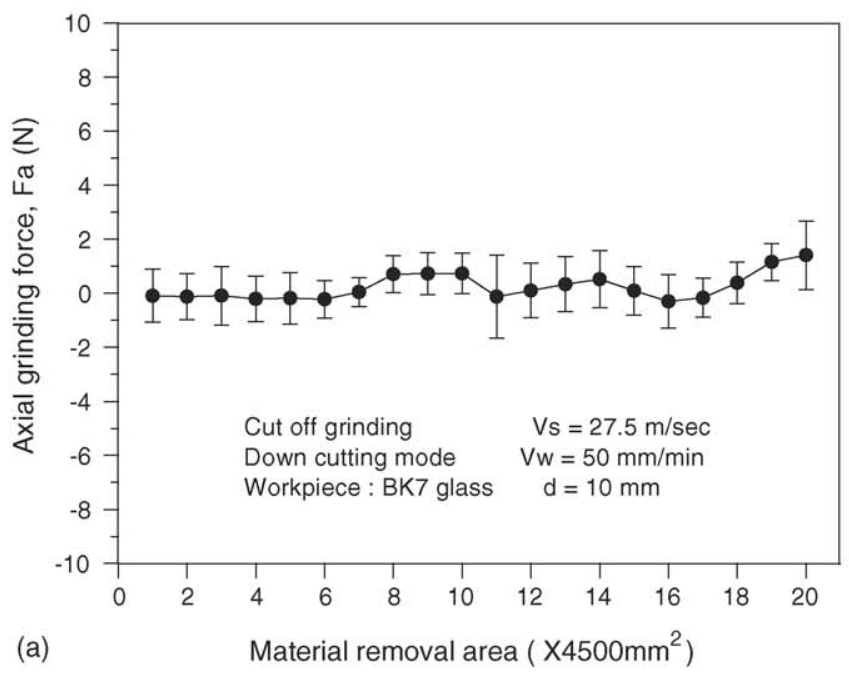

with the increasing of the material removal area. When the wheels at a faster transverse velocity of 100 or $150 \mathrm{~mm} / \mathrm{min}$ were employed to cut-off BK7 glass, the average axial grinding forces produced were a little larger variation (Fig. $6 \mathrm{~b}$ and c). The reason for this may be that the diamond abrasives on the worn wheel produce some macro-fracture and the wheel wear during the cut-off BK7 glass increases. In addition, the average normal grinding forces at a transverse velocity of $50 \mathrm{~mm} / \mathrm{min}$ displayed a stable variation and the average tangential grinding forces showed a little increase. When the transverse velocity was increase to $100 \mathrm{~mm} / \mathrm{min}$, the variation of average normal forces with the increase of the material removal area was smaller than those of a transverse velocity of $50 \mathrm{~mm} / \mathrm{min}$ and the average tangential grinding forces showed a relative low and stability. This causes the grinding ratio produced for $V_{\mathrm{W}}$ of 50 and $100 \mathrm{~mm} / \mathrm{min}$ to obtain a similar state. However, for the transverse velocity of $150 \mathrm{~mm} / \mathrm{min}$, the variation of average normal and tangential forces produced during the cut-off showed the unstable conditions (Fig. 7c). This condition was the results of the diamond abrasive pattern on the worn wheel (refer to Fig. 4c).

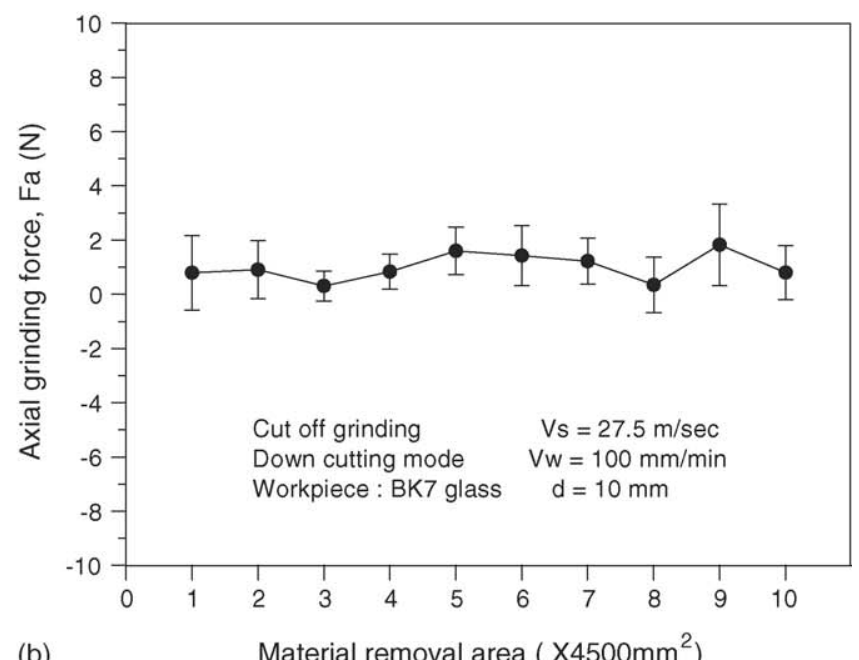

(b)

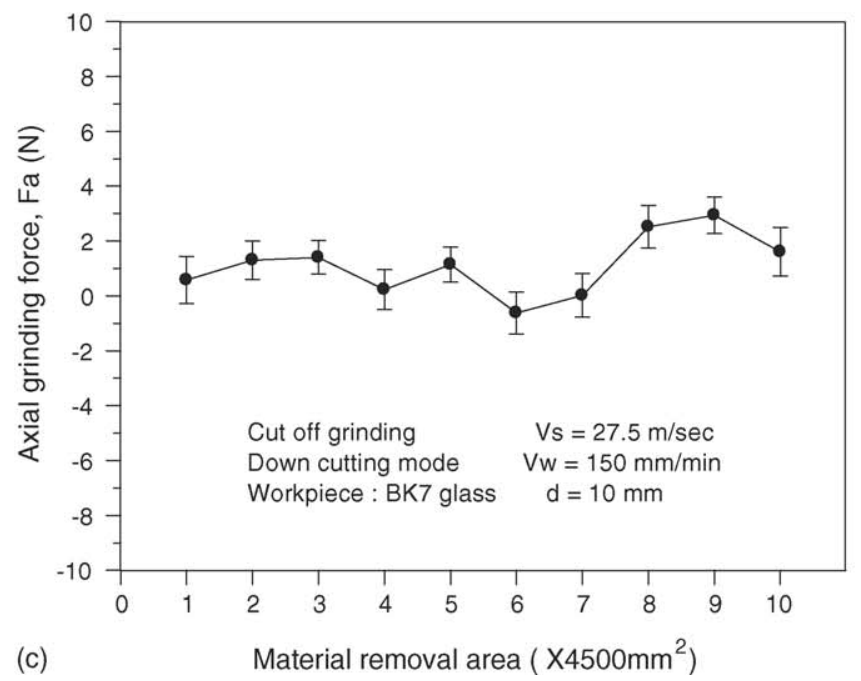

Fig. 6. Variations of the average axial grinding forces, $F_{\mathrm{a}}$, with the material removal area during the cut-off of BK7 glass under three different transverse velocities of: (a) $50 \mathrm{~mm} / \mathrm{min}$, (b) $100 \mathrm{~mm} / \mathrm{min}$ and (c) $150 \mathrm{~mm} / \mathrm{min}$. 

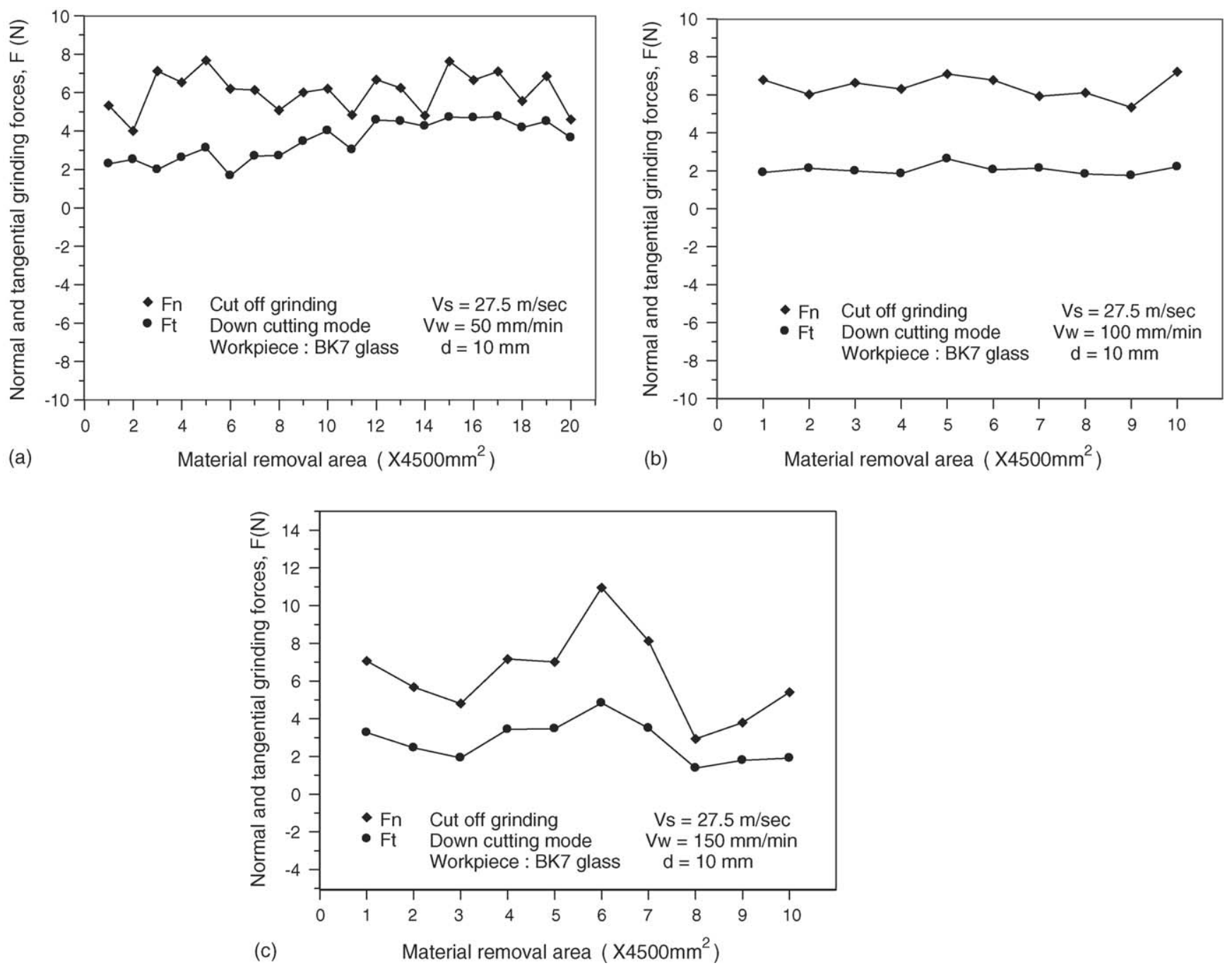

Fig. 7. Variations of the average normal and tangential grinding forces, $F_{\mathrm{n}}$ and $F_{\mathrm{t}}$, produced with the material removal area under three different transverse velocities of: (a) $50 \mathrm{~mm} / \mathrm{min}$, (b) $100 \mathrm{~mm} / \mathrm{min}$ and (c) $150 \mathrm{~mm} / \mathrm{min}$.

This larger force variation will cause the poor cut quality of BK7 glass.

\subsection{Cut-off straightness}

Straightness of cut of BK7 glass obtained at the transverse velocity of $50 \mathrm{~mm} / \mathrm{min}$ during the cut-off grinding as shown in Fig. 8a displays the positive values, which represent the convex conditions of cut profile. The convex amount of the cut profile increased to maximum with the increase of the cut distance, and when the wheel will depart the workpiece, convex values produce the decrease. The average maximum deviation of cut straightness produced in the workpiece was about $100 \pm 25 \mu \mathrm{m}$, which located at the end site of 0.9 times of the workpiece length. This is because the thin diamond wheel cuts initially to produce the unstable state and a lower clamping force subjected by the workpiece's edges. In addition, the frictional time between the side of the thin wheel and the workpiece was longer due to a lower transverse velocity. These phenomena cause the front site of the workpiece to be cut more material, thereby forming a bell shape. When the wheel will depart the workpiece, the wheel was also the more unstable state, which the cut width became larger. Hence, the cut width of two ends removed was larger, which the workpiece's profile displayed a convex shape. When the transverse velocity of cut-off was increased to $100 \mathrm{~mm} / \mathrm{min}$, the average maximum straightness of the cut produced was increased to about $150 \pm 50 \mu \mathrm{m}$ at a $0.5-0.7$ times of the workpiece length as shown in Fig. 8b. The reason for this was that the wheel under a higher transverse velocity produced an initial larger vibration to have a high run-out, which causes a poorer cut straightness than that of transverse velocity of $50 \mathrm{~mm} / \mathrm{min}$. Furthermore, the transverse velocity of cut-off was employed to $150 \mathrm{~mm} / \mathrm{min}$, the straightness of the cut produced displayed a concave or convex deviation and their values' range were about -100 to $100 \mu \mathrm{m}$. Causing this result was possibly that the wheel at a large transverse velocity entered initially into the workpiece to produce toward one side of cut. In addition, a poor straightness of cut during the cut-off grinding is also strongly affected by the overhang length of the cutting edge of the wheel. 

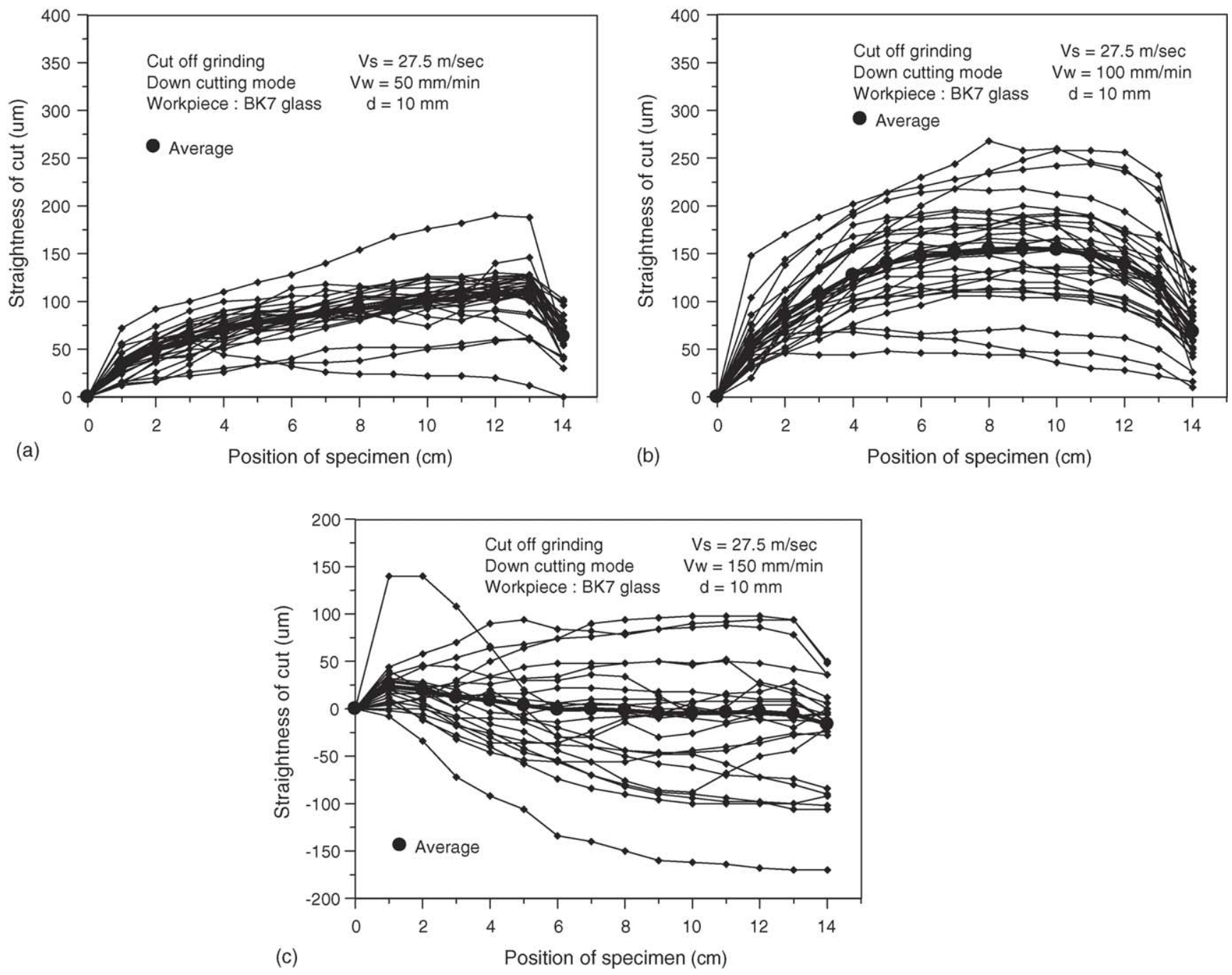

Fig. 8. The straightness of cut obtained during the cut-off grinding of BK7 glass at the transverse velocity of: (a) $50 \mathrm{~mm} / \mathrm{min}$, (b) $100 \mathrm{~mm} / \mathrm{min}$ and (c) $150 \mathrm{~mm} / \mathrm{min}$.

\subsection{Chipping of the cutting edges}

The typical appearance of the sliced surface during the cutoff grinding of BK7 glass as shown in Fig. 9 shows a lot of cracks with the roughness of about $R_{\mathrm{a}} 0.4-1.0 \mu \mathrm{m}$. Fig. 10a and $\mathrm{b}$ shows the appearance of the chipping at the top and bottom cutting edges during the cut-off BK7 glass, respectively, and their chipping sizes are displayed in Fig. 11. From Fig. 10, it can be seen that the bottom of cutting edges showed a larger chipping than the top edges. This was possibly that the down cutting mode was used to produce a downward cutting force and a weak support at the bottom. The chipping size of the bottom produced at a transverse velocity of 50 and $100 \mathrm{~mm} / \mathrm{min}$ was about 620 and $500 \mu \mathrm{m}$, respectively. These conditions were related with smaller tangential cut-off forces and variations (refer to Fig. 7b). When the transverse velocity of cut-off was increased to $150 \mathrm{~mm} / \mathrm{min}$, the chipping size of the bottom obtained was about $600 \mu \mathrm{m}$. In addition, the chipping sizes of the top cutting edges was smaller than those of the bottom edges, and their values were about $300-400 \mu \mathrm{m}$. This was due to the compressive forces at the top cutting edges. The difference of chipping size

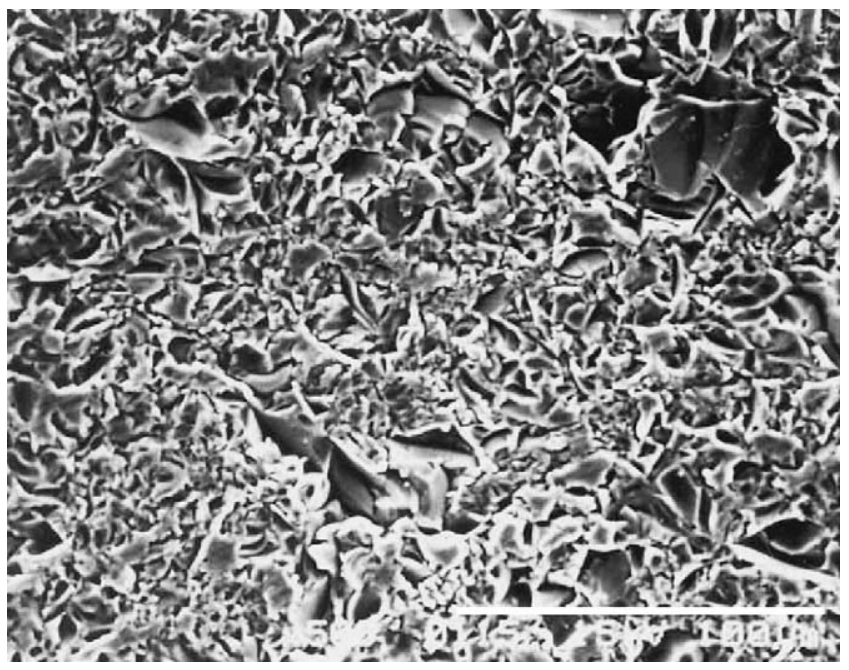

Fig. 9. The typical appearance of the sliced surface during the cut-off grinding of BK7 glass. 

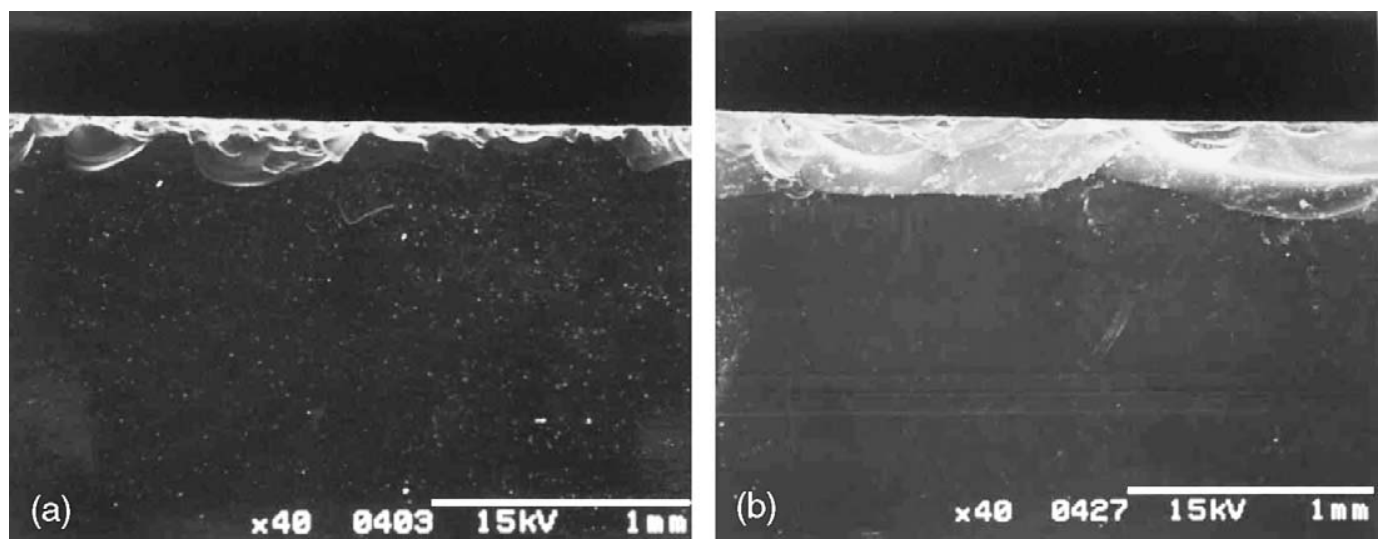

Fig. 10. The typical appearances of the chipping at the (a) top and (b) bottom cutting edges during the cut-off grinding of BK7 glass.

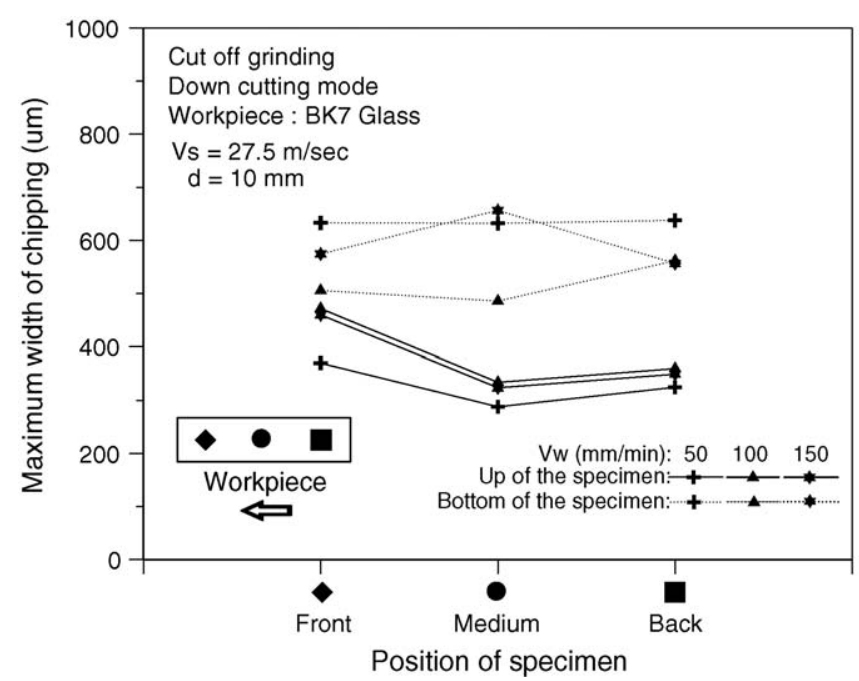

Fig. 11. The maximum width of chipping size on the different position of the up and bottom of the workpiece after the cut-off BK7 glass at the different transverse velocity.

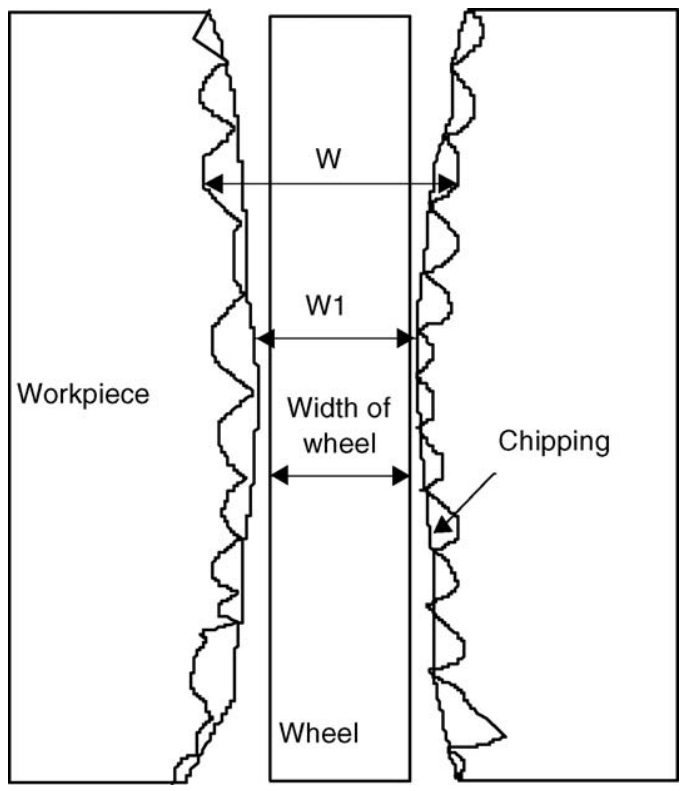

Fig. 12. Scheme of the width of cut, $W_{1}$, total characteristic width of cut, $W$, and chipping produced during the cut-off grinding of BK7 glass.

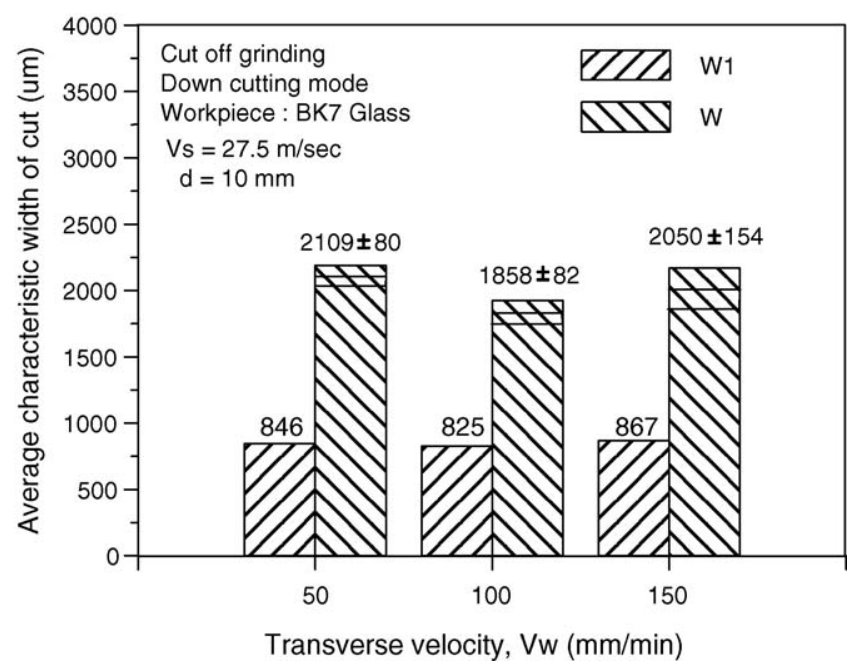

Fig. 13. The average width of cut, $W_{1}$, and the average total characteristic width of cut, $W$, with the confidence of $95 \%$ at the different transverse velocity.

at the different transverse velocity of cut-off was attributed to the variation of cut-off forces. The more the cut-off forces were, the larger chipping sizes were.

The width of cut and chipping produced during the cutoff grinding of BK7 glass are schematically shown in Fig. 12. The total characteristic width of cut, $W$, equaled to the addition of the width of cut and two times of chipping size ( $W=W_{1}+2 \times$ chipping size). The chipping of the cut edges was a significant effect for obtaining the high quality of cut-off. The average width of cut, $W_{1}$, and the average total characteristic width of cut, $W$, with the confidence of $95 \%$ are shown in Fig. 13 . It can be seen that the average total characteristic width of cut, $W$, at a transverse velocity of $100 \mathrm{~mm} / \mathrm{min}$ was $1858 \mu \mathrm{m}$, and it was smaller than those at a transverse velocity of 50 and $150 \mathrm{~mm} / \mathrm{min}$. The reason for this was due to the more stable cutting forces (refer to Fig. 7).

\section{Conclusions}

The worn wheel surface produced at a slower transverse velocity during the cut-off of BK7 glass showed a larger number of protrusive particles and partial breakage particles, which 
display a more effective material removal rate and a good selfredressing behavior. It caused the thin diamond wheel to have a smaller wear and a high grinding ratio. The grinding forces produced at a lower transverse velocity showed a little variation with the increase of material removal area. The straightness of cut during the cut-off of BK7 glass was better. In addition, the wear pattern of the diamond abrasives on the worn wheel exhibited the principal cycle of protrusive particle, partial breakage particle, particle pulled-out and new protrusive particle. Conversely, when a too high transverse velocity was employed, the diamond particles produced on the worn wheel surface displayed a higher percentage of the partial breakage and breakage conditions and the number of the effective new protrusive grits produced less than that of the breakage particle. This causes a larger variation of the grinding forces and a higher wheel wear. Besides, the width of cut and chipping size of the cutting edges produced by the thin blade at a lower or faster transverse velocity showed a larger state due to an unstable vibration. The straightness of cut during the cut-off BK7 glass produced the maximum convex location to move toward the initial site of the cut with the increase of the transverse velocity. However, when the transverse velocity was too high, the straightness of cut obtained was toward uncertainty side during the cut-off grinding of BK7 glass.

\section{Acknowledgments}

The authors are thankful to the National Science Council of the Republic of China for supporting this study under contract
NSC-89-2218-E-211-002. The Taiwan Diamond Industrial Co. (TDC) is also acknowledged for supporting this work.

\section{References}

[1] M. Yamasaka, M. Fujisawa, T. Oku, M. Masuda, Improvement of straightness in precision cut-off grinding using thin diamond wheels, Ann. CIRP 39 (1) (1990) 333-336.

[2] Y. Ohbuchi, N. Ueda, M. Touge, T. Matsuo, K. Takeuchi, S. Hata, High precision cut-off grinding of electronic ceramics with thin diamond wheel, in: International Conference on Precision Engineering, Taiwan, 1997, pp. 391-395.

[3] T. Miwa, I. Inasaki, Blade wear and wafer chipping in dicing processes, in: International Conference on Precision Engineering, Taiwan, 1997, pp. 397-402.

[4] T. Takahashi, P.D. Funkenbusch, Micromechanics of diamond composite tools during grinding of glass, Mater. Sci. Eng. A285 (2000) 69-79.

[5] I. Inasaki, Grinding of hard and brittle materials, Ann. CIRP 36 (2) (1987) 463-471.

[6] H.K. Tonshoff, W.V. Schmieden, I. Inasaki, W. Konig, G. Spur, Abrasive machining of silicon, Ann. CIRP 39 (2) (1990) 621-635.

[7] S.W. Webb, W.E. Jackson, Analysis of blade forces and wear in diamond stone cutting, Trans. ASME J. Eng. Ind. 120 (1998) 84-92.

[8] K. Subramanian, S. Ramanath, M. Tricard, Mechanisms of material removal in the precision production grinding of ceramics, Trans. ASME J. Manuf. Sci. Eng. 119 (1997) 509-519.

[9] S.Y. Luo, Y.S. Liao, C.C. Chou, J.P. Chen, Analysis of the wear of a resin-bonded diamond wheel in the grinding of tungsten carbide, J. Mater. Process. Technol. 69 (1997) 289-296.

[10] J.L. Metzger, Superabrasive Grinding, Butterworths, London, 1986.

[11] S. Malkin, Grinding Technology: Theory and Applications of Machining with Abrasives, Ellis Horwood Limited, England, 1989. 